\title{
Humane endpoints for animals used in training
}

\author{
Jeremiah Dunlap
}

As a trainer in the biomedical research field, I find it gratifying to observe the mounting attention on the training of investigative and animal care personnel. Training in biomedical research has a depth that surprises many people, and I feel there is room for more discussion of how research personnel can be instructed in working with animals while assuring that the animals used in training and instruction receive top-quality care and humane treatment. For example, we need to consider how frequently an individual animal can be used, at what point it should be euthanized and how the different training techniques available influence the frequency of use and selection of endpoints.

In a recent survey on the Laboratory Animal Welfare Training Exchange listserv, a small group of trainers remarked upon the usage of training animals and implementation of humane endpoints in their animal care and use programs. All the respondents used mice and rats in training, and some also housed guinea pigs, hamsters and rabbits for training purposes.

The survey asked three questions: what species are available for training at your institution? How do you set an endpoint for training animals (e.g., by age, by total number of procedures performed, a combination of the two)? Are there any procedures that an animal can undergo only once before being euthanized? (If yes, please elaborate.)

According to one respondent, each mouse or rat could be used in a training session no more than once per week; another of those surveyed mentioned that mice and rats could be used in a maximum of seven sessions and that potentially painful procedures (e.g., intraperitoneal injections) were limited to two per session. Other respondents had different standards for rodents used in their training programs, including an age limit of 6-12 months, after which the rodents would be euthanized or adopted out of the program, if possible.

The results of this short survey indicated that there is not widespread agreement among animal research programs regarding endpoints for animals used in training. One commonality was the special consideration given to procedures that are potentially painful or invasive, restricting the number of these procedures that an individual animal can undergo.

The training protocol at Northwestern University includes a set of standards for rodent species that are not covered by the US Department of Agriculture. Procedures are classified into one of four categories, ranging from Category 1 (including non-invasive techniques such as handling and restraint) to Category 4 (including techniques deemed invasive or high risk such as intracardiac blood collection). Animals can undergo a maximum of five Category 2 techniques, which can be followed by a single Category 3 or a single Category 4 technique. A particular animal can be used in training only once every 2 weeks. At the completion of the final training session, the animal will be euthanized immediately. For any nonsurvival procedures requiring anesthesia, the animal will be euthanized prior to anesthetic recovery. If at any time during or after training sessions an animal exhibits signs of pain or distress, the animal will be further assessed. Euthanasia will occur immediately, if needed to ensure the welfare of the animal.
All training sessions are documented at the cage level including the date, category of training and technique.

These standards are meant to ensure that none of Northwestern University's training rodents is subjected to an unacceptable amount of procedural manipulation, taking into account the total number of procedures performed on each rodent, the type of procedures performed and the frequency with which they occur. Although we can note some similarities between Northwestern University's standards and the endpoints described by the survey respondents, we can also note substantial differences between the two in areas where information is lacking. There is much variety in the approach to humane endpoints for animals used in training from one research program to the next. Since members of different research programs are left to make their own best interpretation, the result is a different approach per program.

The consideration we extend to our training animals is more than just a testament to our commitment to topnotch animal care. Establishing humane endpoints for animals used in training, and facilitating discussions on those endpoints, is another avenue for trainers in our field to encourage personnel to focus on animal welfare. Training can encompass more than the correct way to perform a technique; it can help shape the lens through which laboratory animals are viewed. By exemplifying such great consideration for laboratory animals involved in training, we can reinforce the truth that good animal care and good science are interwoven, that the one contributes to the other. 